\title{
The influence of interface roughness on electrical transport in nanoscale metallic multilayers
}

\author{
D. Aurongzeb and M. Holtz ${ }^{\mathrm{a})}$ \\ Department of Physics, Texas Tech University, Lubbock, Texas 79409 \\ J. M. Berg \\ Department of Mechanical Engineering, Texas Tech University, Lubbock, Texas 79409
}

A. Chandolu and H. Temkin

Department of Electrical Engineering, Texas Tech University, Lubbock, Texas 79409

(Received 26 January 2005; accepted 8 August 2005; published online 23 September 2005)

\begin{abstract}
We study the effects of annealing on the electrical resistivity of thin metallic multilayers of nickel and aluminum. Resistivity is seen to rise with anneal temperature. Above a specific temperature the resistivity decreases. We model this behavior based on the interface evolution due to the formation of intermetallic nanocrystals. The rise is attributed to interface roughening and to constraints placed on the electron mean-free paths by the nanocrystals. At high temperatures, the lateral coalescence preserves the smooth stratification producing the observed drop in film resistivity. (c) 2005 American Institute of Physics. [DOI: 10.1063/1.2058172]
\end{abstract}

\section{INTRODUCTION}

Intermetallics of aluminum and nickel have drawn considerable attention due to their technological importance in several areas. These include corrosion and oxidation resistant coatings, ${ }^{1}$ preparation of materials by self-propagating hightemperature synthesis, ${ }^{2,3}$ and in metallization for microelectronics. ${ }^{4,5}$ Materials with dimensions on the nanometer scale can be used to create inhomogeneous systems forming a class of "artificial solids." ${ }^{, 6}$ Structured systems include self-assembled nanodots and wires, ${ }^{7,8}$ granular and porous media, ${ }^{6,9}$ and ordered layers ${ }^{10,11}$ thus spanning a wide range of physics in one, two, and three dimensions. The resulting properties are strongly influenced by morphology providing tunability of electrical, magnetic, elastic, and optical properties. ${ }^{12}$

Electrical transport through artificially structured materials is of general interest to such areas as magnetotransport and electronic materials. ${ }^{6,10,13,14}$ When considering transport in thin films, several important aspects must be taken into account. First, scattering by impurities and grain boundaries may limit the electron mean-free path $(\lambda)$ in metals. ${ }^{13} \mathrm{Sec}$ ond, transport is quasi-two-dimensional when layer thickness is comparable to or smaller than the bulk value of $\lambda .{ }^{15,16}$ Third, surface and interface roughness can lead to elevated scattering during transport. Each of these effects results in diminished conductivity $(\sigma)$ compared to the analogous ideal bulk material. The effects of surface ${ }^{17}$ and interface ${ }^{14,18}$ roughness on resistivity $(\rho=1 / \sigma)$ in thin metallic films, as well as annealing-induced amorphization, ${ }^{19}$ have received extensive study. However, the effects of interfaces on transport, when they are degraded through controlled formation of nanocrystals, have not been studied. In multilayers, interdiffusion can take place during deposition, under ambient con-

\footnotetext{
a) Author to whom correspondence should be addressed; electronic mail: Mark.Holtz@ttu.edu
}

ditions, and when high-temperature annealing is performed. Formation of alloy nanocrystals at the interfaces is a kinetically driven process. The presence of these nanocrystals will change the transport due to their resistivities, which are generally different from those of the starting materials, and due to increased interface roughness.

We examine the effects the intermetallic nanocrystals have on the resistivity when they are formed at the interfaces in a nickel/aluminum multilayer stack. Metallic multilayers, with thickness on the order of $10 \mathrm{~nm}$, provide a venue for modifying morphology in a controlled fashion and studying its effects on electrical transport. This thickness regime is interesting because it is near the crossover where layer thickness causes intralayer transport to be quasi-two-dimensional. ${ }^{15}$ Furthermore, for thin films the presence of interface roughness is critical in conduction because it enters as a relative quantity. When annealed at temperatures below the melting point of either the $\mathrm{Ni}$ or $\mathrm{Al}$, interdiffusion creates nanocrystals of intermetallic nickel aluminides at the layer interfaces. Previous studies of $\mathrm{Ni} / \mathrm{Al}$ (Ref. 20) and $\mathrm{Nb} / \mathrm{Al}$ (Ref. 21) interdiffusion emphasized high-resolution transmission electron microscopy (TEM) and scanning calorimetry. Nanocrystals initially form at the interfaces, as seen in the TEM images, ${ }^{20}$ and with sufficient anneal time and temperature eventually consume the starting materials. X-ray reflectivity (XRR) measurements, in combination with scanning electron microscopy (SEM), have recently shown that the interface roughness of the multilayers exhibits a striking behavior with annealing. ${ }^{22}$ Below a certain temperature range the layer properties are well defined. As temperature is increased, the interfaces rapidly roughen as an aggregate of $\mathrm{Ni}$ and $\mathrm{Al}$, and the interdiffusion products form. Remarkably, above these temperatures the layer morphology is preserved due to the faster growth along the interfaces, which causes the nanocrystals to laterally coalesce. The layer roughness is well described adapting a kinetic growth model in which the nanocrystals grow both along and perpendicular 


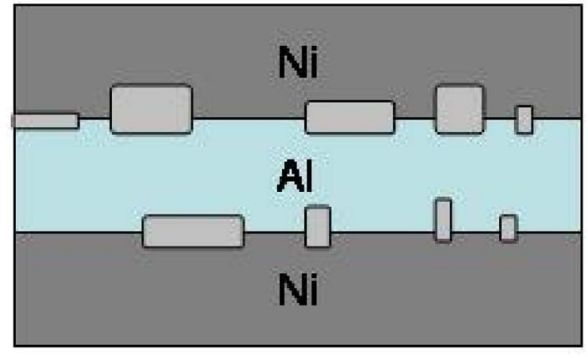

FIG. 1. Depiction of nickel and aluminum multilayers following the formation of nickel aluminide nanocrystals at the interface.

to the interfaces, as depicted in Fig. 1. This model accurately describes the interface roughening observed over a narrow anneal-temperature range. ${ }^{22}$ We extend here the nanocrystal formation model developed in Refs. 20-22 to describe the experiments on the $\mathrm{Ni} / \mathrm{Al}$ multilayer resistivity. Upon annealing, $\rho$ is found to rise rapidly above a specific temperature followed by a significant reduction at higher temperature. We develop a model demonstrating that interface scattering is the key factor in describing the observed resistivity dependence.

\section{EXPERIMENTAL DETAILS}

Equally thick layers of pure $\mathrm{Ni}$ and $\mathrm{Al}$ were deposited on glass substrates using electron-beam evaporation with base pressures of $\leqslant 10^{-7}$ Torr. Substrate temperatures rise $<50{ }^{\circ} \mathrm{C}$ in situ above room temperature during deposition. Multiple layer samples (up to 20 periods) were deposited with periods $L=20$ and $50 \mathrm{~nm}$. Annealing was carried out for $10 \mathrm{~min}$ in a conventional furnace with nitrogen atmosphere to minimize oxidation of the Ni surface layer. Multilayer films were studied using X-ray diffraction and XRR (Philips $\mathrm{X}$-Pert, $\mathrm{Cu} K \alpha$ source) ${ }^{23}$ atomic force microscopy, and SEM cross sections. Resistivities were obtained at room temperature using a four-point probe. SEM reveals the expected stratification of the deposited layers (Fig. 2 inset), with layer thickness in agreement with the in situ deposition monitor.

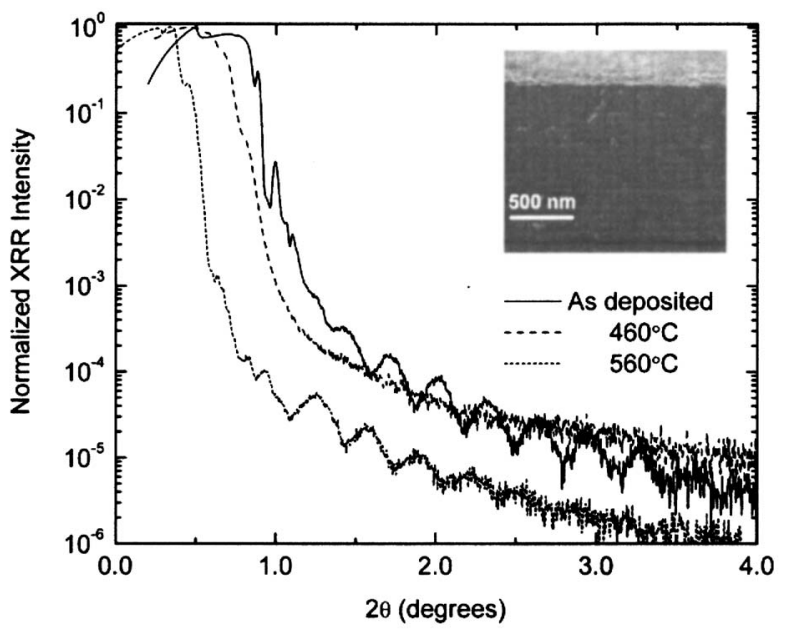

FIG. 2. XRR measurements of $L=50 \mathrm{~nm} \mathrm{Ni/Al} \mathrm{multilayers} \mathrm{annealed} \mathrm{at}$ different temperatures. Data are normalized (only) for clarity. Note the disappearance of fringes at $460{ }^{\circ} \mathrm{C}$. Inset: SEM cross section of the asdeposited multilayers.
XRR data in Fig. 2 show multiple interference fringes due to the multilayer structure. As described in Ref. 22, the fringes vanish in a narrow anneal-temperature range due to roughening arising from the formation of intermetallic nanocrystals at the interfaces. For the $L=50 \mathrm{~nm}$ case, this temperature range is from 380 and $480{ }^{\circ} \mathrm{C}$, while for $L=20 \mathrm{~nm}$ the roughening happens in a lower temperature range, from 300 to $400{ }^{\circ} \mathrm{C}$. As deposited, we see two distinct critical angles in Fig. 2, which we attribute to $\mathrm{Ni}$ and Al. Estimates of the material densities from these critical angles are in agreement with the accepted values for $\mathrm{Ni}$ and $\mathrm{Al}$. Annealing at $360^{\circ} \mathrm{C}$ $(L=50 \mathrm{~nm})$ causes these angles to coalesce. Following anneals above $460{ }^{\circ} \mathrm{C}$ (but below the melting point of $\mathrm{Al}$ ) we observed two critical angles, which are very close to each other. We attribute these effects to the evolution of the multilayer stack as intermetallic compounds form at the layer interfaces, as described below.

\section{INTERFACE ROUGHNESS AND NANOCRYSTAL FORMATION}

The layer thickness, along with interface and surface roughness values are obtained through a full dynamic XRR simulation using a commercial grazing incidence $\mathrm{X}$-ray analysis (GIXA, Philips) program based on Ref. 24. Parameters that varied in the analysis are thicknesses of the $\mathrm{Ni}$ and $\mathrm{Al}$ layers and the interface and surface roughness values. The material densities also enter the simulations but not as variable parameters. In Fig. 3(a) we show interface roughness at each anneal temperature obtained from analysis of the XRR measurements. The as-deposited films have interface roughness of $1.7 \pm 0.2 \mathrm{~nm}$. This is attributable to both grain formation and slight interdiffusion of $\mathrm{Ni}$ and $\mathrm{Al}$ which take place during and post deposition. ${ }^{2}$ Disappearance of the XRR fringes is attributed to interface roughening. Also shown in Fig. 3(a) are surface roughness values extracted from the XRR analysis and measured directly using atomic force microscopy (AFM). We see a slight rise in surface roughness over the full anneal range studied, in contrast to the interface roughness dependence. It is possible that the interface roughness also varies between the interfaces of a given thickness and the following annealing. This would particularly be the case for the near-surface layers. We have allowed the interface roughness to vary in fitting several data sets but find variation to be $<10 \%$ of the interface roughness. Thus, adding this level of complexity to the fitting does not sufficiently improve our results to warrant including it in the procedure, and we ignore gradients in the interface roughness in the remainder of our analysis.

Figure 3 also shows the bilayer resistivity (scaled per bilayer) for (b) $L=20$ and (c) $L=50 \mathrm{~nm}$ measured versus anneal temperature. Resistivity values of the as-deposited layers agree with what is expected for thin films of $\mathrm{Ni}$ and $\mathrm{Al}$ using bulk values for their conductivities. ${ }^{18}$ In both cases we find $\rho$ to remain comparable to that of the as-deposited $\mathrm{Ni} / \mathrm{Al}$ multilayer at low temperatures, then to rise rapidly for higher anneal temperature. The rise for the $L=50 \mathrm{~nm}$ multilayers occurs at slightly higher temperature than for $L=20 \mathrm{~nm}$ 

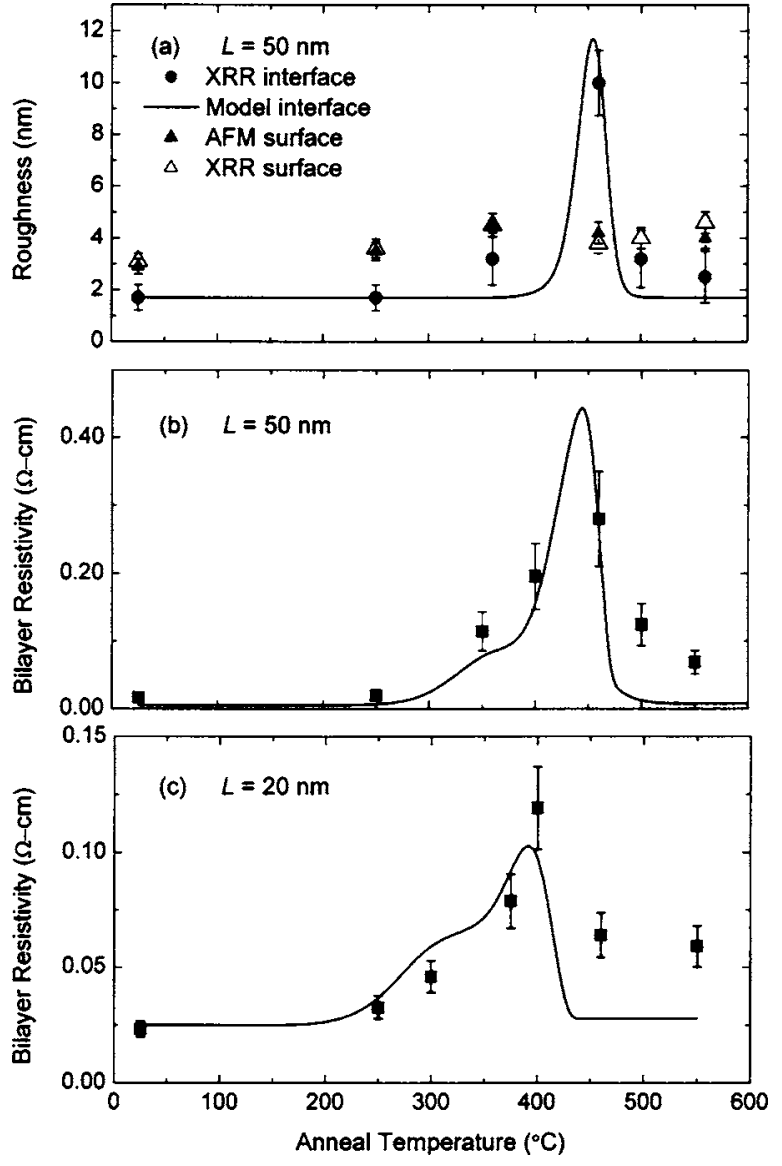

FIG. 3. (a) Interface $(\bullet)$ and surface $(\triangle)$ roughness from $X R R$, and surface roughness from AFM $(\mathbf{\Lambda})$ measurements vs anneal temperature. Measured resistivity scaled per bilayer $\left(N \rho_{\text {meas }}\right)$ vs anneal temperature for multilayers with periods (b) $50 \mathrm{~nm}$ and (c) $20 \mathrm{~nm}$. The solid curves are model calculations accounting for the interface roughness and nanocrystal domain size. The calculations require no arbitrary scaling.

films. At higher temperature, the resistivities exhibit maxima followed by a noticeable decrease. We now develop a model describing the observed behavior.

Nanocrystal formation in the $\mathrm{Ni} / \mathrm{Al}$ layers is driven by interdiffusion. As described in Refs. 20 and 22, the growth takes place at the layer interfaces. For simplicity, domains are considered to be cylindrical. The average lateral or radial domain growth rate (i.e., parallel to the interface plane) is given by

$$
\frac{d r}{d t}=K_{\perp} \exp \left(-E_{\perp} / k T\right)
$$

and the average vertical growth rate is

$$
\frac{d h}{d t}=\frac{1}{h} K_{\|} \exp \left(-E_{\|} / k T\right)
$$

Activation energies $E_{\perp}=2.0 \mathrm{eV}$ and $E_{\|}=1.49 \mathrm{eV}$ and growth prefactors $\left(K_{i}\right)$ are taken from Ref. 22. From this model, we obtain the average vertical and radial domain sizes following high-temperature anneal steps. For convenience, we define the radial fill factor

$$
f_{A}=1-\exp \left(-n \pi r^{2}\right),
$$

which is used to quantify the lateral coalescence of nanocrystals. Statistical values of $r, h$, and $f_{A}$ depend on anneal temperature and duration. Quantity $n$ in Eq. (3) is the average areal density of the nanocrystals. ${ }^{20}$ The root-mean-square (rms) interface roughness $\delta$ is obtained for an average domain height $h$ according to

$$
\delta^{2}-\delta_{0}^{2}=f_{A}\left(1-f_{A}\right) h^{2},
$$

where $\delta_{0}$ is the native roughness. ${ }^{22}$ The calculated curve shown in Fig. 3(a) is $\delta$ versus anneal temperature for which $f_{A}$ and $h$ are calculated using Eqs. (1)-(3). Agreement with the data is good indicating that the anneal-induced interface roughness observed using XRR is described by the kinetic interdiffusion model. The radial growth produces a quasitwo-dimensional layer of either $\mathrm{Al}$ or $\mathrm{Ni}$, which is interrupted, in the low-density limit, by isolated nanocrystals with $h$ and $r<L / 2$. Thus, a conduction path exists through the $\mathrm{Al}$, say, and the resistivity will be close to that of the starting layer. As the nanocrystals grow, they begin to coalesce. Eventually, only the interdiffused end product will be present and the transport will be dominated by the resistivity of that material.

\section{RESISTIVITY}

We consider the effects of nanocrystal formation on the resistivity of $\mathrm{Ni} / \mathrm{Al}$ multilayer films through the interface roughness. In order to account for the interface roughness, an approach based on the Boltzmann transport equation ${ }^{18}$ is adapted to include a phenomenological term ${ }^{25}$ which modifies the bilayer resistivity according to

$$
\rho_{\text {int }}=\left(\frac{4 \delta}{L}\right) \tanh \left(\frac{L}{4 \delta}\right) \rho_{\mathrm{nc}},
$$

where $\rho_{\mathrm{nc}}$ is the resistivity of the interface nickel aluminide nanocrystals. For $\rho_{\text {nc }}$ we use the approach of Ref. 26 in which the conductivity of a metal composed of domains is

$$
\sigma_{\text {nc }}=\sigma_{\text {bulk }}\left[1-1.5 \alpha+3 \alpha^{2}-3 \alpha^{3} \ln (1+1 / \alpha)\right] .
$$

Here, $\sigma_{\text {bulk }}$ is for the bulk material from which the individual domains are formed and

$$
\alpha=\frac{\lambda R}{b(1-R)} .
$$

In this expression, $R \approx 0.5$ is the reflection coefficient of electrons by the interface between two domains and $b$ is the separation between the domains $(\sim 1 \mathrm{~nm})$. Values of $\mathrm{R}$ found in the literature are typically from 0.1 to $0.5,{ }^{27}$ although values as high as 0.9 (Ref. 28) have been reported. Two distinct length regimes are important. ${ }^{12,15}$ For domains larger than the electron mean-free path, $\alpha$ in Eq. (7) is limited by $\lambda$. However, for domains smaller than or on the order of the bulk electron mean-free path, $\lambda$ is limited by the domain size. This is the case for our multilayers, where the bulk Drude value of $\lambda$ is much larger than the domain sizes. ${ }^{16} \mathrm{We}$ apply this approach to the effect of nanocrystal domain formation using the kinetic model in Eqs. (1) and (2) for do- 


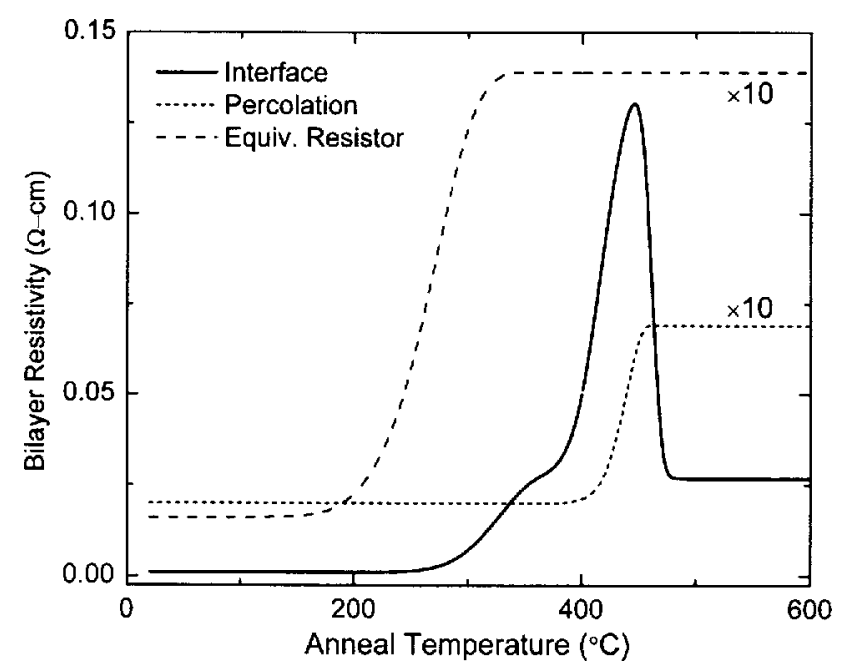

FIG. 4. Calculations of the effects the different morphologies have on the bilayer $(50 \mathrm{~nm})$ resistivity with anneal temperature. Solid curve is the effect of interface roughness on resistivity. Also shown are the percolation model and the laterally coalesced equivalent (parallel) layer resistor.

main size and limit $\lambda$ in Eq. (7) according to the minimum of the lateral domain size or intrinsic mean-free path. Our x-ray diffraction measurements show that $\mathrm{NiAl}$ and $\mathrm{NiAl}_{3}$ are the dominant nickel aluminides formed for the $L=50$ and $20 \mathrm{~nm}$ multilayers, respectively. ${ }^{22,29}$ The bulk conductivity of the nanocrystalline materials are $1.89 \times 10^{4} \Omega^{-1} \mathrm{~cm}^{-1}$ (Ref. 30) for $\mathrm{NiAl}$ and $6.6 \times 10^{4} \Omega^{-1} \mathrm{~cm}^{-1}$ for $\mathrm{NiAl}_{3}$ (Ref. 31). We thus obtain the anneal-temperature dependence of the resistivity as the interface roughness grows, as shown in Fig. 4 below $\sim 450{ }^{\circ} \mathrm{C}$. The initial rise with anneal temperature is primarily due to the increase in $\rho_{\mathrm{nc}},{ }^{32}$ while the second rise stems from the growth in interface roughness. The calculated rms roughness (normalized) is also shown in Fig. 4.

Above $450{ }^{\circ} \mathrm{C}(L=50 \mathrm{~nm})$, smooth interfaces are maintained upon annealing. ${ }^{22}$ However, conduction is primarily through the nickel aluminides formed upon annealing in contrast to conduction through $\mathrm{Ni}$ or $\mathrm{Al}$ at lower temperatures. With this role reversal, interfaces in the nickel aluminide multilayers are smooth at higher anneal temperature, as seen in the rms roughness trend in Fig. 3(a). The two critical angles seen in the XRR data in this anneal-temperature range are consistent with the presence of two materials. This diminishing interface roughness produces a reduction in $\rho_{\text {int }}$ using Eq. (5), which is graphed in Fig. 4. Either $\rho_{\mathrm{Ni}}$ or $\rho_{\mathrm{Al}}$ may be used for $\rho_{\mathrm{nc}}$ since the calculated curve is not sensitive to this choice. The high-temperature result tends to the NiAl value.

In Figs. 3(b) and 3(c) we compare the calculated model dependence with the measured $\rho$ with no scaling. The model utilizes Eqs. (1)-(7) in order to obtain an analytic dependence and does not directly rely on the interface roughness values obtained experimentally from XRR. Excellent agreement with the data is seen in three important regards. First, the rise in resistivity, followed by a reduction at high anneal temperature, is well described by combining the effects of interface roughness and nanocrystal formation. Second, the calculated curves reproduce adequately the dependence on bilayer period of the temperature at which the elevated resis- tivity occurs. Third, the rise in $\rho$ begins at low temperature. This latter rise is not present in our model if we only take the interface roughness into account. That is, the effect of domain size on $\lambda$ is also essential in describing the observed dependence.

We have also considered alternative approaches to calculating the resistivity of the $\mathrm{Ni} / \mathrm{Al}$ multilayers with anneal temperature. Conceptually, percolation theory lends itself to this analysis. ${ }^{33}$ If we view the starting layers as a highly conductive material $\sigma_{0}$ and the end product as a poorer conductor $(\sigma \sim 0)$, we can apply the percolation model which describes a transition between conductor and insulator when domains reach a critical fill factor, $p=p_{c}$. Conductivity varies according to $\sigma=\sigma_{0}\left(p-p_{c}\right)^{\gamma}$ for $p>p_{c}$ and is zero otherwise. We use $p_{c}=0.6$ for the percolation threshold and $\gamma=1$ for the critical exponent in the quasi-two-dimensional case. In the current context, $p=1-f_{A}$ from Eq. (3) and the conductivity of the nickel aluminide end product must be used rather than $\sigma=0$. Figure 4 shows the temperature dependence of $\rho$ obtained using the percolation approach and the kinetic growth model. The calculated rise in $\rho$ is abrupt and occurs at a higher temperature than the experimentally observed increases. The magnitude of the increase is much smaller than what is needed to agree with the data and no decrease in resistivity is obtained. This is due to the sharpness seen in the transition of $f_{A}$ with anneal temperature. We note that the dependence is not sensitive to the exact values used for $p_{c}$ and $\gamma$ due to the abruptness in $f_{A}$. We conclude that the observed dependence in $\rho$ of our films is not described by the percolation approach.

We have also considered a sandwich multilayer stack in which the $\mathrm{Ni}$ and $\mathrm{Al}$ in one bilayer transforms into four layers upon annealing: nickel, nickel aluminide, aluminum, and a second layer of nickel aluminide. With anneal, the fraction of nickel aluminide in the bilayer grows at the expense of the $\mathrm{Ni}$ and $\mathrm{Al}$. Using our kinetic equations, we calculate the equivalent parallel bilayer resistance versus anneal temperature as shown in Fig. 4. We use a similar resistivity calculation as that described in Ref. 19 for $\mathrm{Ni}-\mathrm{Zr}$ layers. The calculated dependence shows a rise at low temperature. However, like the percolation model this parallel resistor approach shows a small increase in resistivity and does not predict the observed decrease in resistivity at high temperature.

\section{CONCLUSIONS}

We have observed an interesting increase followed by a decrease of the resistivity of $\mathrm{Ni} / \mathrm{Al}$ bilayers with anneal temperature. The dependence is attributed to the formation of intermetallic nanocrystals at the interfaces. At low temperature, interface roughness is low so that $\rho$ remains constant. As nanocrystals form, the roughness increases and the resistivity rises by a factor of $\sim 20$. At higher temperatures, lateral coalescence of the nanocrystals preserves the layer smoothness and a drop in the resistivity is observed. We note also that the temperature range over which the rise observed in $\rho$ depends on the bilayer period, an attribute predicted by our 
model. We conclude that lateral transport in metallic multilayers is strongly sensitive to both interface roughness and nanocrystal domain formation.

\section{ACKNOWLEDGMENT}

The authors acknowledge National Science Foundation Grant Nos. CTS-0210141 and ECS-0323640 and the J. F. Maddox Foundation for support of this research.

${ }^{1}$ L. A. Marvina and V. B. Marvin, Tech. Phys. 42, 49 (1997).

${ }^{2}$ A. J. Gavens, D. van Heerden, A. B. Mann, M. E. Reiss, and T. P. Weihs, J. Appl. Phys. 87, 1255 (2000).

${ }^{3}$ M. Monagheddu, N. Bertolini, P. Giuliani, C. Zanotti, and U. Anselmi Tamburini, J. Appl. Phys. 92, 594 (2002).

${ }^{4}$ J. W. Mayer and S. S. Lau, Electronic Materials Science: Integrated Circuits in Silicon and GaAs, (MacMillan, New York, 1990).

${ }^{5}$ X. He, K. Tao, and Y. Fan, J. Vac. Sci. Technol. A 14, 2564 (1996)

${ }^{6}$ C. H. Arns, M. A. Knackstedt, and K. R. Mecke, Phys. Rev. Lett. 91, 215506 (2003)

${ }^{7}$ C. -H. Chiu, Phys. Rev. B 69, 165413 (2004).

${ }^{8}$ S. Lepadatu and Y. B. Xu, Phys. Rev. Lett. 92, 127201 (2004).

${ }^{9}$ I. S. Beloborodov, K. B. Efetov, A. V. Lopatin, and V. M. Vinokur, Phys. Rev. Lett. 91, 246801 (2003).

${ }^{10}$ M. Tsoi, V. Tsoi, J. Bass, A. G. M. Jansen, and P. Wyder, Phys. Rev. Lett. 89, 246803 (2002).

${ }^{11}$ A. E. Meyerovich and I. V. Ponomarev, Phys. Rev. B 67, 165411 (2003).

${ }^{12}$ L. Chico, M. P. Lopez-Sancho, and M. C. Munoz, Phys. Rev. B 65, 184429 (2002)

${ }^{13}$ G. Reiss, J. Vancea, and H. Hoffmann, Phys. Rev. Lett. 56, 2100 (1986).

${ }^{14}$ P. M. Levy, S. Zhang, and A. Fert, Phys. Rev. Lett. 65, 1643 (1990).

${ }^{15}$ M. Gurvitch, Phys. Rev. B 34, 540 (1986).
${ }^{16}$ C. Reale, J. Phys. F: Met. Phys. 4, 2218 (1974).

${ }^{17}$ Z. Tesanovic, M. V. Jaric, and S. Maekawa, Phys. Rev. Lett. 57, 2760 (1986).

${ }^{18}$ A. Misra, M. F. Hundley, D. Hristova, H. Kung, T. E. Mitchell, M. Nastasi, and J. D. Embury, J. Appl. Phys. 85, 302 (1999).

${ }^{19}$ J. Eckert, L. Schultz, and K. Urban, J. Mater. Res. 6, 1874 (1991).

${ }^{20}$ E. Ma, C. V. Thompson, and L. A. Clevenger, J. Appl. Phys. 69, 2211 (1991).

${ }^{21}$ K. R. Coffey, K. Barmak, and D. A. Rudman, J. Appl. Phys. 72, 1341 (1992).

${ }^{22}$ D. Aurongzeb, M. Holtz, M. Daugherty, J. Berg, A. Chandolu, J. Yun, and H. Temkin, Appl. Phys. Lett. 83, 5437 (2003).

${ }^{23}$ L. Grave de Peralta and H. Temkin, Appl. Phys. Lett. 93, 1974 (2003).

${ }^{24}$ L. G. Parratt, Phys. Rev. 95, 359 (1954).

${ }^{25}$ R. Skomski, M. Emrech, and J. M. D. Coey, Nanostruct. Mater. 1, 337 (1992).

${ }^{26}$ A. F. Mayadas and M. Shatzkes, Phys. Rev. B 1, 1382 (1970).

${ }^{27}$ X. Zhang, M. F. Hundley, A. Malinowski, A. Misra, H. Wang, and M. Nastasi, J. Appl. Phys. 95, 3644 (2004)

${ }^{28}$ X. Jun, Y. Zhou, C. O. Kim, Y. P. Lee, H. Xu, and S. K. Gong, J. Appl. Phys. 91, 6071 (2002).

${ }^{29}$ U. Rothhaar, H. Oechsner, M. Scheib, and R. Müller, Phys. Rev. B 61, 974 (2000)

${ }^{30}$ X. Y. Qin, L. D. Zhang, B. M. Wu, M. L. Tian, Y. L. Du, D. S. Yang, and L. Z. Cao, J. Appl. Phys. 80, 4776 (1996).

${ }^{31}$ J. B. Dunlop, G. Grüner, and A. D. Caplin, J. Phys. F: Met. Phys. 4, 2203 (1974).

${ }^{32} \mathrm{We}$ have also examined the role of $\mathrm{Ni}-\mathrm{Al}$ eutectics which may form at the interfaces. Including these contribute slightly to the initial rise in resistivity providing a small shift toward lower temperature, but do not describe the overall rise and fall trends observed. See R. Simoneau and G. Bégin, J. Appl. Phys. 44, 1461 (1973).

${ }^{33}$ N. T. Liang, Y. Shan, and S. Wang, Phys. Rev. Lett. 37, 526 (1976). 